\title{
Correction to: Incidence and predictive factors of postoperative hypocalcaemia according to type of thyroid surgery in older adults
}

\author{
María Paz Barbier $^{1} \cdot$ Evelin Mingote $^{1} \cdot$ Noelia Sforza $^{1} \cdot$ Yanina Morosán Allo ${ }^{1} \cdot$ Maximiliano Lotartaro $^{2}$. \\ Leonardo Serrano ${ }^{2}$ - María Pía Fossati ${ }^{3}$ - Tomas Meroño ${ }^{4,5}$. Cristina Faingold ${ }^{1}$. Claudia Sedlinsky ${ }^{1}$. \\ Gabriela Brenta $\mathbb{D I}^{1}$
}

Published online: 26 November 2021

(c) Springer Science+Business Media, LLC, part of Springer Nature 2021

Correction to: Endocrine https://doi.org/10.1007/s12020-021$02840-9$

In the original version of this article, the Last Name should appear before the first name for the article to be found in any search (e.g.: Pubmed) or be cited.

The last name is now underlined to be identified from the first name :

Barbier María Paz,

Mingote Evelin,

Sforza Noelia,
Morosán Allo Yanina,

Lotartaro Maximiliano,

Serrano Leonardo,

Fossati María Pía,

Meroño Tomas,

Faingold Cristina,

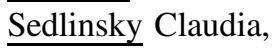

Brenta Gabriela,

The original article has been revised

Gabriela Brenta

gbrenta@gmail.com

1 Servicio de Endocrinología y Metabolismo, Unidad Asistencial Dr. César Milstein, Buenos Aires, Argentina

2 Servicio de Cirugía General, Unidad Asistencial Dr. César Milstein, Buenos Aires, Argentina

3 Laboratorio Central, Unidad Asistencial Dr. César Milstein, Buenos Aires, Argentina

4 Laboratorio de Biomarcadores y Nutrimetabolómica,

Departamento de Nutrición, Ciencias de la Alimentación y Gastronomía, Red de Referencia en Tecnología de Alimentos (XaRTA), Instituto de Nutrición y Seguridad Alimentaria (INSA), Facultad de Farmacia y Ciencias de la Alimentación, Universidad de Barcelona, Barcelona, Spain

5 CIBER de Fragilidad y Envejecimiento Saludable (CIBERFES), Instituto de Salud Carlos III, Madrid, Spain 